\title{
Analysis of Electrical Dipoles Interaction Forces as a Function of the Distance and of the Form of Electrical Force Law
}

\author{
Ioan Has' ${ }^{1}$, Simona Miclaus ${ }^{1}$, Aurelian Has ${ }^{2}$ \\ ${ }^{1}$ Land Forces Academy, Sibiu, Rm. Valcea, Romania \\ ${ }^{2}$ Independent Researcher, Rm. Valcea, Romania \\ Email:simo.miclaus@gmail.com, hasaurelian@yahoo.com, hasavo@yahoo.com
}

How to cite this paper: Has, I., Miclaus, S. and Has, A. (2018) Analysis of Electrical Dipoles Interaction Forces as a Function of the Distance and of the Form of Electrical Force Law. Journal of Applied Mathematics and Physics, 6, 1886-1895.

https://doi.org/10.4236/jamp.2018.69160

Received: August 20, 2018

Accepted: September 17, 2018

Published: September 20, 2018

Copyright $\odot 2018$ by authors and Scientific Research Publishing Inc. This work is licensed under the Creative Commons Attribution International License (CC BY 4.0).

http://creativecommons.org/licenses/by/4.0/ Open Access

\begin{abstract}
Here, we initially introduced and demonstrated two principles: orientation OR principle and attraction AT principle of electrical dipoles. The OR principle stipulates that any two electrical dipoles $P 1 A, P 1 B$, from two bodies $A$ and $B$, at any distance in the free state each, will be reciprocally oriented parallel and in the same sense if the electrical interaction forces $F$ between them are of decreasing type with distance $r$. If the electrical interaction forces $F$ are of increasing type with distance, the two dipoles will be reciprocally oriented parallel but on the opposite sense. The AT principle stipulate that any two electrical dipoles $P 1 A, P 1 B$, at any distance in the free state each, will present always a reciprocal force of attraction $F_{D}$ in both cases of orientation accordingly to OR principle in case of any type of electrical force $F$ decreasing or increasing with distance. These findings may complete our previous work where we found that $F_{D}$ force, between two electrical dipoles $P 1 A, P 1 B$ considered at atomic and nuclear level, is in fact the actual gravitation Newton force $F_{N}$. The paper must be considered together with this work for more consistency.
\end{abstract}

\section{Keywords}

Electrical Forces, Completed Coulomb's Law, Electric Dipoles Interactions, Torsion Moment of Orientation, Attraction Force between Two Dipoles Is Gravitation

\section{Observations about Actual Explanation for the Absence of Electrical Interactions at Long Distances}

Today, it is largely admitted that at long distances, at astronomical scales, at 
planetary scale and even at building scales, the electrical forces $F_{C}$ given by classical Coulomb's law of electrical forces:

$$
F_{C}=\frac{q_{1} q_{2}}{4 \pi \varepsilon_{0} r^{2}}=\frac{k q_{1} q_{2}}{r^{2}} ; \text { with } k=1 / 4 \pi \varepsilon_{0} ;
$$

between two neutral bodies are negligible and should not be taken into account, as it happens today.

The only forces considered today at such long distances are the gravitational forces $F_{N}$ given by classical Newton's law.

$$
F_{N}=-G \frac{M m}{r^{2}}
$$

This affirmation excludes the electromagnetic phenomena which consist of more complex combinations of electric and magnetic forces originated at atomic scales.

But it is unlikely that the interaction forces acting between two electrical charges $q_{1}, q_{2}$ may be perfectly described by mathematical simple laws as in (1), at any distances $r$, and of any scales, in the micro and macro universe.

At this point of our analysis, the above affirmation is justified by the reality complexity or its infinity and also by discrepancies between calculus results of actual simple laws from (1), (2) and some experimental measurements and observations concerning these forces, reported in the past.

Discrepancies concerning the Coulomb's law are confirmed by modern experiments, which allow scientists to conclude that in the case of very short distances (such as between molecules, atoms, nuclei, particles) the law (1) is not precisely correct [1], but also it has not yet been proven in the case of long distances [2]. In such circumstances, we can conclude that the Coulomb's law in the current form (1) is not perfect and that it can be improved.

For a more accurate description of the infinite reality, including ether presence which will be considered throughout this paper, as we previously demonstrated [3] [4] [5] [6], the most appropriate (but not singular mathematical) mode of electrical forces description, will be probable, a long or infinite series of successive powers of the $1 / r$ distance.

In the present, it is accepted that macroscopic bodies have a random distribution of $+q$ and $-q$ charges, which will generate a random distribution of dipoles in any direction, and all coefficients $k_{i}$ from (3) are canceled [7].

$$
\phi_{M}=\frac{k_{0}}{\varepsilon_{0} r}+\frac{k_{1}}{\varepsilon_{0} r^{2}}+\frac{k_{2}}{\varepsilon_{0} r^{3}}+\cdots
$$

This means that starting from small distances from any neutral body to infinite distances, the influence of the electric charges from that body is considered today to be null. But we will show below that this approach isn't correctly justified. 


\section{Our New Hypothesis and Principles, Concerning the Dipole Interactions at Long Distances}

\subsection{Justification of Introducing the Two New Principles of Electric Dipoles Interaction, $\mathrm{OR}$ and AT}

Despite the fact that bodies $A, B \ldots$, are neutral, the $+q$ and $-q$ charges are in fact always separated in space at atomic and nuclear scale, and consequently every charge $+q$ or $-q$ will exert its electrical interaction force $F_{C}$ to infinite distance according to (1) or to a corrected Coulomb law as in [8] [9] [10] [11].

So in nature practically all the matter must be regarded as being organized always as electrical dipoles $P 1, P 2, P 3 \ldots$ as result of Coulomb law action even at smallest scales.

We will show next, that neglecting electrical forces and their potential even for distant bodies is not correct because we can admit and demonstrate the following two principles:

a) OR principle of the reciprocal orientation of distant dipoles.

b) AT principle of the permanent attraction of oriented distant dipoles.

\subsection{Presentation and Justification of OR Principle}

In order to demonstrate the OR principle, we can calculate the torsion moments $+M$ (clockwise) and $-M$ (counterclockwise) created by an oriented dipole $P 2$ from body B upon an un-oriented dipole $P 1$ (disposed at $90^{\circ}$ in body A).

In this situation (Figure 1) we can distinguish two cases, depending on the variation mode with distance $r$, of interaction Coulomb's forces $F$ (reference frame $\mathrm{xOy}$ is attached to A body).

Case 1a). of decreasing $F$ forces. Firstly, for this calculus, we suppose the interaction forces $F_{a}$ (attraction for $+q /-q$ charges when $F_{a}<0$, according to (1)) and $F_{r}$ (rejection for $+q /+q$ or $-q /-q$ charges, $F_{r}>0$, according to (1)) between electrical charges $+q$ and $-q$ as being some vectors $F$ of central orientation decreasing with the increasing distance $r \gg l$, between body $A$ and body $B$ according to the law of the general type (4), generalizing (1), with $n$ a natural number:

$$
F=\frac{q_{1} q_{2}}{4 \pi \varepsilon_{0} r^{n}}=\frac{k q_{A} q_{B}}{r^{n}}
$$

including the simple case $n=1$ :

$$
F=\frac{k q_{A} q_{B}}{r}
$$

Product $q_{A} \cdot q_{B}$ from (4), (4a) comprises for simplicity, also coefficients $k / 4 \pi \varepsilon_{0}$. Utilizing (4a), we obtained from Figure 1 the clockwise torsion moment $+M$ and counterclockwise $-M$ moment, given upon $P 1$ dipole, by forces $F$ from $P 2$ dipole, as follows:

$$
M=F \frac{l}{2}=m\left(q_{A} q_{B}\right)
$$




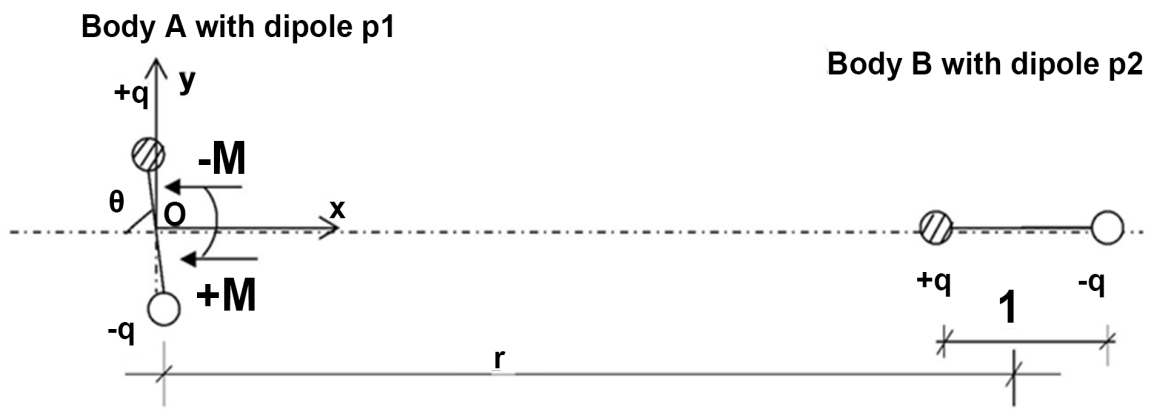

Figure 1. Situation of two distant dipoles P1 and P2 from bodies A and B, which manifest a reciprocal orientation with $\mathrm{xOy}$ frame attached to $\mathrm{A}$ body.

where function $m$ represents the components of total $M$ moments, given by various pairs of $q$ charges.

$$
\begin{aligned}
+M & =m\left(q_{A}^{+} q_{B}^{-}\right)+m\left(q_{A}^{-} q_{B}^{-}\right)=k q^{2}\left[\left(\frac{1}{r+l / 2}\right) \cdot\left(\frac{l}{2}\right)+\left(\frac{1}{r+l / 2}\right) \cdot\left(\frac{l}{2}\right)\right] \\
& =k q^{2} \frac{2}{r+l / 2} \cdot \frac{l}{2}=k q^{2} \frac{l}{r+l / 2} \\
-M & =m\left(q_{A}^{+} q_{B}^{+}\right)+m\left(q_{A}^{-} q_{B}^{+}\right)=k q^{2}\left[\left(\frac{1}{r-l / 2}\right) \cdot\left(\frac{l}{2}\right)+\left(\frac{1}{r-l / 2}\right) \cdot\left(\frac{l}{2}\right)\right] \\
& =k q^{2} \frac{2}{r-l / 2} \cdot \frac{l}{2}=k q^{2} \frac{l}{r-l / 2}
\end{aligned}
$$

The ratio $r_{M}$ of the two moments results from (6) and (7):

$$
r_{M}=\frac{+M}{-M}=\frac{k q^{2} \frac{l}{r+l / r}}{k q^{2} \frac{l}{r-l / r}}=\frac{r-l / 2}{r+l / 2}<1 ; \rightarrow \rightarrow-M>+M ;
$$

This result of $M$ moments ratio $r_{M}$ indicates that the orientation of dipole $P 1$ will be in the same sense/direction as dipole $P 2$, in this case of the decreasing positive forces $F$.

Case $1 b)$. of decreasing $F$ forces and sign change. We now must calculate what happens in the hypothetical case when the forces $F$ from (4) and (4a) will change the sign from positive $+F$ to negative $-F$ forces, including the simple case $n=1$ :

$$
F=-\frac{k q_{A} q_{B}}{r}
$$

In this modified Case 1b), we admit hypothetically, an inversion of the actual physical rule (attraction force $-F_{a}$ between $+q$ and $-q$ charges), becoming repulsion force $+F_{r}$ between $+q$ and $-q$ charges and attraction force $-F_{a}$ between $+q$ and $+q$ or between $-q$ and $-q$ charges.

In this modified Case 1b) the Equations (6) and (7) becomes, considering the same senses of rotation of $+M$ and $-M$ moments, as in Figure 1: 


$$
\begin{aligned}
+M & =m\left(q_{A}^{+} q_{B}^{+}\right)+m\left(q_{A}^{-} q_{B}^{+}\right)=k q^{2}\left[\left(-\frac{1}{r-l / 2}\right) \cdot\left(\frac{l}{2}\right)+\left(-\frac{1}{r-l / 2}\right) \cdot\left(\frac{l}{2}\right)\right] \\
& =-k q^{2} \frac{2}{r-l / 2} \cdot \frac{l}{2}=-k q^{2} \frac{l}{r-l / 2} \\
-M & =m\left(q_{A}^{+} q_{B}^{-}\right)+m\left(q_{A}^{-} q_{B}^{-}\right)=k q^{2}\left[\left(-\frac{1}{r+l / 2}\right) \cdot\left(\frac{l}{2}\right)+\left(-\frac{1}{r+l / 2}\right) \cdot\left(\frac{l}{2}\right)\right] \\
& =-k q^{2} \frac{2}{r+l / 2} \cdot \frac{l}{2}=-k q^{2} \frac{l}{r+l / 2}
\end{aligned}
$$

The ratio $r_{M}$ of the two moments results:

$$
r_{M}=\frac{+M}{-M}=\frac{-k q^{2} \frac{l}{r+l / r}}{-k q^{2} \frac{l}{r-l / r}}=\frac{r-l / 2}{r+l / 2}<1 ; \rightarrow \rightarrow-M>+M
$$

This result of the $M$ moments ratio $r_{M}$ indicates that the orientation of dipole $P 1$ is in the same sense/direction as dipole $P 2$, also in the case of inverse sign of decreasing forces $F$ (as in Case 1a). of decreasing actual forces $F$ ).

So we conclude that changing the sign of $F$ force, have no influence upon the orientation rule of dipoles $P 1$ and $P 2$.

Case $2 \mathrm{a})$. of increasing $F$ forces. Secondly, for this calculus, we suppose the interaction force $F_{a}$ (attraction) and $F_{r}$ (rejection) between electrical charges $q$ as being some vectors of central orientation increasing with the distance $r \gg l$, according to the laws of the general type (13), with $n$ a natural number:

$$
F=k_{1} q_{A} \cdot q_{B} \cdot r^{n}
$$

including the simple case $n=1$ or the limit power case of $\ln r$, in both cases $F$ force increasing with $r$.

$$
F=k_{1} q_{A} \cdot q_{B} r \quad \text { or } \quad F=k_{1} q_{A} \cdot q_{B} \ln r
$$

Utilizing (13b) we obtain from Figure 1:

$$
\begin{aligned}
+M & =m\left(q_{A}^{+} q_{B}^{-}\right)+m\left(q_{A}^{-} q_{B}^{-}\right)=k_{1} q^{2}\left[\left(r+\frac{l}{2}\right) \cdot\left(\frac{l}{2}\right)+\left(r+\frac{l}{2}\right) \cdot\left(\frac{l}{2}\right)\right] \\
& =k_{1} q^{2} 2\left(r+\frac{l}{2}\right) \cdot \frac{l}{2}=k_{1} q^{2}\left(r+\frac{l}{2}\right) \cdot l \\
-M & =m\left(q_{A}^{+} q_{B}^{+}\right)+m\left(q_{A}^{-} q_{B}^{+}\right)=k_{1} q^{2}\left[\left(r-\frac{l}{2}\right) \cdot\left(\frac{l}{2}\right)+\left(r-\frac{l}{2}\right) \cdot\left(\frac{l}{2}\right)\right] \\
& =k_{1} q^{2} 2\left(r-\frac{l}{2}\right) \cdot \frac{l}{2}=k_{1} q^{2}\left(r-\frac{l}{2}\right) \cdot l
\end{aligned}
$$

The ratio $r_{M}$ of the two moments results:

$$
r_{M}=\frac{+M}{-M}=\frac{k_{1} q^{2}\left(r+\frac{l}{2}\right) \cdot l}{k_{1} q^{2}\left(r-\frac{l}{2}\right) \cdot l}=\frac{r+\frac{l}{2}}{r-\frac{l}{2}}>1 ; \rightarrow \rightarrow+M>-M ;
$$


This result of $M$ moments ratio $r_{M}$ indicates that the orientation of dipole $P 1$ is in the opposite sense/direction as dipole $P 2$, in case of increasing positive forces.

In Case $2 \mathrm{~b}$ ), of increasing $F$ forces and sign change, when the force $F$ is also as increasing with $r$ type, but of reversed sign, is similar to the Case $1 \mathrm{~b}$ ). And hence the result of the calculus of $r_{M}$ ratio will be similar: indicating that the orientation of dipole $P 1$ is in the opposite sense/direction as dipole $P 2$. (as in Case 1b). in this case of increasing positive forces $F$.

And so at points Case 1) and Case 2), the OR principle was demonstrated.

The OR principle stipulate that in case of decreasing electrical forces $F$ with $r$ the orientation of dipole $P 1$ is in the same sense/direction as dipole $P 2$ irrespective of sign of $F$.

But in case of increasing electrical forces $F$ with $r$, the orientation of dipole $P 1$ is in the opposite sense/direction as dipole $P 2$ irrespective of sign of $F$.

\subsection{Presentation and Justification of AT Principle}

In order to demonstrate the AT principle, will be calculated forwards the resultant force $R$, appearing between two oriented dipoles, $P 1 A$ and $P 1 B$, according to OR principle, given by forces of attraction $F_{a}$ and by forces of repulsion $F_{r}$ existing between $+q$ and $-q$ charges, as in actual $F_{\mathrm{C}}$ or completed $F$, Coulomb's law.

It must determine if the force $R$ is the attraction or repulsion force, as a function of the form of the electric force law $F$.

The situation of two dipoles $P 1 A$ and $P 1 B$ originated in A body and in B body respectively, identically oriented and equal to each other, with polar moment value $r=q \cdot l$, is presented in Figure 2. The two possible cases of the $F$ force variation with the distance $r$ between two electric charges will be analyzed below: Case 1) the decreasing variation and Case 2) the increasing variation of $F$ force with $r$.

But we must remind that first calculus concerning the attraction force $F_{a}$ and the rejection force $F_{r}$ between two farther electrical dipoles was performed by us in [8] [11], but without presenting there the significance of the result of that calculus, consisting in the sign of resultant force $R$.

Also there in [8] [11], was made a simplification of calculus by introducing a substitution: $\alpha=k q_{A} q_{B}$.

But here we will emphases that such result, will conduct at a new important principle, the AT principle of permanent attraction of electric dipole, described firstly here below.

In order to better understand the origin of $\mathrm{AT}$ principle we will resume here without the above substitution, the calculus of forces $F_{a}$ and of $F_{r}$ from [8] [11] performed in the two cases, Case 1 and Case 2, of the $F$ force variation with the distance $r$.

Case 1). of decreasing $F$ forces. Firstly, we suppose the interaction force $F_{a}$ (attraction) and $F_{r}$ (rejection) between electrical charges $+q$ and $-q$ as being some vectors of central orientation decreasing with the distance $r \gg l$, according to the laws of the general type, from Equations (4) or (4a). 


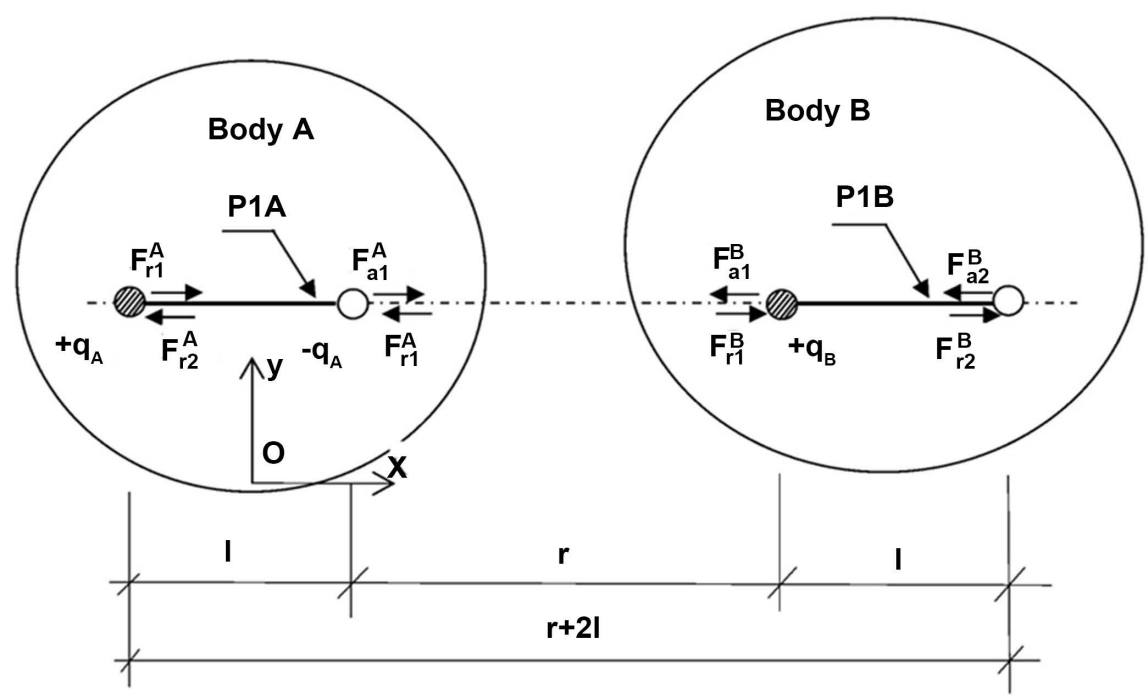

Figure 2. Forces $\mathrm{F}$ acting between a pair of identical oriented dipoles P1A and P1B considered at atomic and nuclear level, from $\mathrm{A}$ and $\mathrm{B}$ bodies with $\mathrm{xOy}$ frame attached to $\mathrm{A}$ body.

From Figure 2, we now can calculate directly by simple algebra, utilizing Equation (4b), the total force upon each dipole, exerted by the other dipole. Here in calculus, we consider the + sign for force values having the sense of real force as in Figure 2, which corresponds to the attraction force $F_{a}\left(F_{a}<0\right)$ and to repulsion force $F_{r}\left(F_{r}>0\right)$, forces exerted by the charges $+q$, $-q$, as in Coulomb law (4).

From Figure 2 notating $-q_{A} \rightarrow q_{A}^{-}$and having $q_{A}^{-} q_{B}^{+}=q_{A}^{+} q_{B}^{-}$, we consider first, the forces exerted upon $P 1 A$ dipole by a $P 1 B$ dipole. Utilizing the simple case (4b) the resultant forces $F_{a}$ and $F_{r}$ values results as follows:

$$
\begin{aligned}
& F_{A}=F_{a 1}^{A}+F_{a 2}^{A}=\frac{k q_{A}^{-} q_{B}^{+}}{r}+\frac{k q_{A}^{+} q_{B}^{-}}{r+2 l}=k q_{A}^{-} q_{B}^{+} \frac{2 r+2 l}{r(r+2 l)} \\
& F_{R}=F_{r 1}^{A}+F_{r 2}^{A}=\frac{k q_{A}^{-} q_{B}^{-}}{r+l}+\frac{k q_{A}^{+} q_{B}^{+}}{r+l}=2 k q_{A}^{-} q_{B}^{-} \frac{1}{(r+l)}
\end{aligned}
$$

If we rewrite the expression from the denominator from (17), one obtains:

$$
r(r+2 l)=(r+l)^{2}-l^{2}
$$

From (19) and admitting $r \gg l$ it results:

$$
r(r+2 l)<(r+l)^{2}
$$

Introducing (20) in (17) and comparing with (18) it results:

$$
\begin{gathered}
\left|F_{a}\right|=\left|k q_{A}^{+} q_{B}^{-} \frac{2 r+2 l}{r(r+2 l)}\right|>\left|k q_{A}^{-} q_{B}^{-} \frac{2 r+2 l}{(r+l)^{2}}\right|=\left|F_{r}\right| \\
\left|F_{a}\right|>\left|F_{r}\right|
\end{gathered}
$$

From (22) it results that in the case of forces $F$ of the type as in Equations (4), (4b) decreasing $(n=+1)$ with the $r$ distance, the attraction force $F_{a}$ will be great- 
er than the repulsion force $F_{r}$ and finally an attraction force $R_{a}=F_{a}-F_{r}>0$ between the two identically oriented dipoles $P 1 A, P 1 B$ results. This result is correct even if the power of $r$ is any $n>1$, as can easily be demonstrated.

Case 2) of increasing F forces. Let's suppose the force $F$ increases with the distance $r$, including a law of the form (13b).

$$
F=k_{1} q_{A} \cdot q_{B} \ln r
$$

with coefficient $k_{1}$ being similar with coefficient $k$ from Equation (4a) but having the appropriate measure units considering that the term $\ln r$ must be adimensional.

Again from Figure 2, for identically oriented dipoles $P 1 A, P 1 B$ (Figure 2), the total forces upon $P 1 A$ dipole utilizing (23) will be:

$$
\begin{aligned}
& F_{a}=F_{a 1}^{A}+F_{a 2}^{A}=k_{1} q_{A}^{-} q_{B}^{+} \ln r+k_{1} q_{A}^{+} q_{B}^{-} \ln (r+2 l)=k_{1} q_{A}^{-} q_{B}^{+} \ln [r(r+2 l)] \\
& F_{r}=F_{r 1}^{A}+F_{r 2}^{A}=k_{1} q_{A}^{-} q_{B}^{-} \ln (r+l)+k_{1} q_{A}^{+} q_{B}^{+} \ln (r+l)=k_{1} q_{A}^{-} q_{B}^{-} \ln (r+l)^{2}
\end{aligned}
$$

Comparing $F$ and $F_{r}$ from Equations (24) and (25), and considering Equation (20) it results:

$$
\begin{gathered}
\left|F_{a}\right|=\left|k_{1} q_{A}^{-} q_{B}^{+} \ln [r(r+2 l)]\right|<\left|k_{1} q_{A}^{-} q_{B}^{-} \ln (r+l)^{2}\right|=\left|F_{r}\right| \\
\left|F_{a}\right|<\left|F_{r}\right|
\end{gathered}
$$

From (27) it results that in the case of increasing $F$ forces of the type from (23), the repulsion force $F_{r}$ between two identically oriented dipoles $P 1 A, P 1 B$, will be greater than the attractive force $F_{a}$ and the resultant force $R_{r}=F_{r}-F_{a}>0$, will be a repulsion force. This result is correct even if the increasing variation has the general form $F=\alpha^{\prime} r^{n}$ with $n>1$, as it can easily be demonstrated.

But in this Case 2 of increasing $F$ forces (23), the natural orientation of two dipoles becomes reverted compared with those from Figure 2, according to the OR principle from Section 2.2., and the interaction force between the two dipoles $P 1 A$ and $P 1 B$ will be again an attraction force $R_{a}=F_{r}-F_{a}<0$ as for decreasing forces.

So, the AT principle stipulate that in case of decreasing electrical forces $F$ with $r$ an attraction force $R_{a}$ between the two identically naturally oriented dipoles $P 1 A, P 1 B$ results.

Also in case of increasing electrical forces $F$ with $r$, because the two dipoles $P 1 A, P 1 B$ will be naturally inverse oriented accordingly to OR principle, AT principle stipulate that again an attraction force $R_{a}$ between the two dipoles $P 1 A$, $P 1 B$ results.

And so, the simultaneous action of OR principle and of AT principle, will give birth of a permanent attraction between two any electrical dipoles irrespective of $F$ force type.

The above analytical calculus and its results, yields the above OR principle and AT principle, whose result must be real.

This effect of permanent attraction of electrical dipoles $P 1 A, P 1 B$ can be observed also in case of a group in any number of usual magnets $m_{i}$ as spheres of 
about $2 \mathrm{~cm}$ radius, which being separated initially, they will tend permanently to gather together as a clew, at a final natural free equilibrium position. Indeed they not separate or move away in space towards an alternate natural equilibrium position in our laboratory scale. Such magnets $m_{i}$ may constitute a correct intuitive physical model of atomic or nuclear electric dipoles.

\section{Conclusions and Consequences}

This article is intended to complete our previous series of articles and conference presentations, concerning the gravitation and ether [8] [9] [10] [11] [12].

Consequently, this paper analysis considered the interactions of electrical charges from the matter, which may really appear at very long distances as terrestrial or astronomical ones, in fact in the range of common gravitational forces. These were logical and in accordance with Coulomb's law (actual or corrected as in [11]) that in the presence of the electrical distinct $+q /-q$ charges acting at an infinite distance, anywhere in space will have some interactions between ever distinct electrical $+q /-q$ charges because always electrical $+q /-q$ charges will be grouped as electrical dipoles $P 1, P 2, P 3 \ldots$

Based also on the above observations from Section 1, we have demonstrated in the current article that no matter the form of electrical force law $F$, and accordingly to OR principle and to AT principle, an attraction dipole force $F_{D}$ will act always between any two distant dipoles, which we have proved in [11] to be even the so-called gravitation Newton force $F_{N}$.

\section{Conflicts of Interest}

The authors declare no conflicts of interest regarding the publication of this paper.

\section{References}

[1] Israelachvili, J. (1991) Intermolecular and Surface Forces. Academic Press, London 7-260.

[2] Luca, E., Ciubotariu, C., Maftei, G., Zet, G., Jeflea, A. and Pasnicu, C. (1996) Fizica, Vol. 2. Editura Stiintifica, Bucharest, 65-70. (In Romanian)

[3] Has, I. (2000) The Analysis of an Alternative Light Path in Michelson's Experiment. Romanian Reports in Physics, 52, 775-789.

[4] Has, I., Miclaus, S. and Has, A. (2010) An Alternative Light Path Analysis in Michelson's Interferometer Experiment. Physics Essays, 23, 248-257. https://doi.org/10.4006/1.3357572

[5] Has, I., Miclaus, S. and Has, A. (2014) A Reanalysis of the Theory of Interferometer Experiment Demonstrating That Michelson's Analysis Contains an Error, Including the Boat Model Analysis, So Readmitting the Ether Presence. Optics, 3, 25-32. https://doi.org/10.11648/j.optics.20140304.11

[6] Has, I., Miclaus, S. and Has, A. (2015) A Reanalysis of the Theory of Interferometer Experiment Demonstrating that Michelson's Analysis Contains an Error, Including the Boat Model Analysis, So Readmitting the Ether Presence. 3rd Annual Interna- 
tional Conference on Chemistry \& Physics, Athens, 20-23 July 2015.

[7] Purcell, E.M. (1972) Electricity and Magnetism. Berkeley physics Course. McGraw-Hill, New York, 59, 318, 328.

[8] Has, I., Miclaus, S. and Has, A. (2008) Analysis of a Possible Correlation between Electrical and Gravitational Forces. Physics Essays, 21, 303-312. https://doi.org/10.4006/1.3038751

[9] Has, I., Miclaus, S. and Has, A. (2011) A Theoretical Confirmation of the Gravitation New Origin, Having a Special Electrical Nature. FQMT11 Conference on Physics, Prague, 26-29 July 2011.

[10] Has, I., Miclaus, S. and Has, A. (2017) Theoretical Confirmation of the Gravitation New Origin Having a Dipolar Electrical Nature with Coulomb's Law Corrected. $2^{\text {nd }}$ International Conference on Physics, Brussels, 28-30 August 2017.

[11] Has, I., Miclaus, S. and Has, A. (2015) A Theoretical Confirmation of the Gravitation New Origin Having a Dipolar Electrical Nature with Coulomb Law. American Journal of Modern Physics, 4, 97-108. https://doi.org/10.11648/j.ajmp.20150403.11

[12] Has, I. and Miclaus, S. (2017) An Initial Model of Ether Describing Electromagnetic Phenomena, Including Gravity. Physics Essays, 30, 45-56.

https://doi.org/10.4006/0836-1398-30.1.45 